\title{
2052. Eigensensitivity of damped system with defective multiple eigenvalues
}

\author{
Pingxin Wang ${ }^{1}$, Xibei Yang ${ }^{2}$ \\ ${ }^{1}$ School of Mathematics and Physics, Jiangsu University of Science and Technology, \\ Zhenjiang, Jiangsu 212003, China \\ ${ }^{2}$ School of Computer Science and Engineering, Jiangsu University of Science and Technology, \\ Zhenjiang, Jiangsu 212003, China \\ ${ }^{2}$ Corresponding author \\ E-mail: ${ }^{1}$ pingxin_wang@hotmail.com, ${ }^{2}$ zhenjiangyangxibei@163.com \\ Received 27 December 2015; received in revised form 12 February 2016; accepted 17 February 2016 \\ DOI http://dx.doi.org/10.21595/jve.2016.15791
}

\begin{abstract}
This paper considers the sensitivity of defective multiple eigenvalues of reducible matrix pencil, the average of eigenvalues is proved to be analytic, the derivatives of the average eigenvalues and the corresponding eigenvector matrices are obtained when the generalized eigenvalue is reducible. The sensitivity of defective multiple eigenvalues of a quadratic eigenvalue problem dependent on several parameters are also obtained by the result of generalized eigenvalue problem. The results are useful for investigating structural optimal design, model updating and structural damage detection.
\end{abstract}

Keywords: sensitivity analysis, generalized eigenvalue problem, defective eigenvalues, quadratic eigenvalue problem.

\section{Introduction}

Matrix eigensensitivity analysis has extensive applications in some engineering applications, for example, structural optimal design [1, 2], model updating [3, 4] and structural damage detection [5]. For example, in sensitivity-based finite element (FE) model updating, the eigensolutions of the analytical model serve to construct the objective function, and the eigensensitivities represent a linearized estimate of the change in the eigenvalues and eigenvectors due to perturbations of the elemental parameters of the FE model. As a result, the study of the variation of the eigenvalues and eigenvectors due to variations in the system parameters, or more precisely the sensitivity of eigensolutions, has emerged as an important area of research.

Most of the early theoretical works, such as [6-10], were concentrated on existence of derivatives of simple eigenvalues and their corresponding eigenvectors of a matrix or matrix pencil. However, in problems such as those of dynamics of symmetric structures, the corresponding matrices can have repeated eigenvalues. The sensitivity analysis of multiple eigenvalues and associated invariant subspaces of a matrix or matrix pencil have been investigated by many researchers [11-15]. More generally, Andrew et al. [16] discussed the existence of derivatives of eigenvalues and corresponding eigenvectors of an analytic matrix-valued function, which includes the quadratic eigenvalue problem considered here as a special case.

In practical numerical computation, a number of methods for the eigenpair derivatives have been developed [17-35], but most of the results are under the condition that the eigenvalues are simple or repeated eignvalues with well-separated derivatives. The requirement that the repeated eigenvalues must have well-separated derivatives was relaxed in Andrew and Tan [36]. The sensitivity of multiple eigenpairs of the quadratic eigenvalue problem are considered in [37-41]. Li et al. [42] showed that the undamped viscously or nonviscously damped eigenproblems can be considered as a degenerated case of general nonlinear eigenproblems and developed an unified eigensensitivity method for both distinct and repeated eigenvalues. Recently Qian et al. [43] extended the methods of [36] for generalized eigenvalue problem to quadratic eigenvalue problem, and proposed numerical methods for computing first and higher order derivatives of multiple eigenpairs of quadratic eigenvalue problem, and obtained a particular solution to the governing equation of the derivatives of eigenvectors by using the QR decomposition or SVD. Some of the 
existing works on sensitivity of eigensolutions of quadratic eigenvalue problem are reviewed by Adhikari in $[44,45]$.

Unfortunately, most of the works of sensitivity analysis of repeated eigenvalues and their corresponding eigenvectors are concentrated on non-defective matrices, although some results of a matrix are available in [14]. This paper aims at developing a method for sensitivity calculation of a defective repeated eigenpairs of generalized eigenvalue and quadratic eigenproblems. The remainder of this paper is arranged as follows. In Section 2, the sensitivity of defective multiple eigenvalues of generalized eigenvalue problem are derived. In Section 3, we focus on dealing with Sensitivity of defective multiple eigenvalues of quadratic eigenvalue problem, and derive the derivatives of the average eigenvalues and the corresponding eigenvector matrices. In Section 4, one example is performed for illustrative purpose. Finally, we make some concluding remarks in Section 5 .

Throughout this paper we use the following notation. $\mathbf{C}^{m \times n}$ denotes the set of complex matrices, $\mathbf{C}^{n}=\mathbf{C}^{n \times 1}, \mathbf{C}=\mathbf{C}^{1} . \mathbf{I}_{n}$ is the identity matrix of order $n, \operatorname{diag}\left(a_{1}, \ldots, a_{n}\right)$ stands for the diagonal matrix with diagonal elements $a_{1}, \ldots, a_{n} . \mathbf{A}^{T}$ denotes the transpose of a matrix $\mathbf{A}$. If $\mathbf{A}=\left(a_{i j}\right) \in \mathbf{C}^{m \times n}, \quad \mathbf{B}=\left(b_{i j}\right) \in \mathbf{C}^{m \times n}$, then $\mathbf{A} \otimes \mathbf{B}=\left(a_{i j} \mathbf{B}\right) \in \mathbf{C}^{m n \times m n}$ denotes the Kronecker product of matrix $\mathbf{A}$ and $\mathbf{B}$.

\section{Sensitivity of defective eigenpairs of reducible generalized eigenvalue problem}

Suppose that $L$ is an open set of $\mathbf{C}^{N}, p=\left(p_{1}, \ldots, p_{N}\right) \in L, \mathbf{A}(p), \mathbf{B}(p) \in \mathbf{C}^{n \times n}$ are analytic matrix-valued functions in some neighborhood $N\left(p^{*}\right)$ of the point $p^{*} \in L$. Without loss of generality we may assume that the point $p^{*}$ is the origin of $\mathbf{C}^{N}$. In this section we study sensitivity analysis of the following generalized eigenvalue problem:

$\mathbf{A}(p) \mathbf{x}(p)=\lambda(p) \mathbf{B}(p) \mathbf{x}(p), \quad \lambda(p) \in \mathbf{C}, \quad \mathbf{x}(p) \in \mathbf{C}^{n}, \quad p \in \aleph(0)$.

Except where stated otherwise, we consider the case in which:

(i) $\{\mathbf{A}(p), \mathbf{B}(p)\}$ is a reducible matrix pencil for $p \in \aleph(0)$, i.e., $B(p)$ is invertible.

(ii) Eq. (1) has an eigenvalue $\lambda_{1}$ with multiplicity $r>1$ when $p=0$ and $\lambda_{1}$ is a defective multiple eigenvalue of the matrix pencil $\{\mathbf{A}(0), \mathbf{B}(0)\}$.

Before giving the main result, we cite some related lemmas.

Lemma 1. [46] Let A, B $\in \mathbf{C}^{n \times n}$ and suppose $\{\mathbf{A}, \mathbf{B}\}$ is a regular matrix pencil, then there exist invertible matrices $\mathbf{P}, \mathbf{Q} \in \mathbf{C}^{n \times n}$, such that:

$\mathbf{P A Q}=\left(\begin{array}{cc}\mathbf{J} & 0 \\ 0 & \mathbf{I}_{n_{2}}\end{array}\right), \quad \mathbf{P B Q}=\left(\begin{array}{cc}\mathbf{I}_{n_{1}} & 0 \\ 0 & \mathbf{N}\end{array}\right)$,

where:

$\mathbf{J}=\operatorname{diag}\left(\mathbf{J}_{1}\left(\lambda_{1}\right), \ldots, \mathbf{J}_{r}\left(\lambda_{r}\right)\right) \in C^{n_{1} \times n_{1}}, \quad \lambda_{i} \neq \lambda_{j} \quad(i \neq j), \quad 1 \leq i, j \leq r$,

$\mathbf{J}_{i}\left(\lambda_{i}\right)=\operatorname{diag}\left(\mathbf{J}_{i}^{(1)}\left(\lambda_{i}\right), \ldots, \mathbf{J}_{i}^{\left(k_{i}\right)}\left(\lambda_{i}\right)\right) \in \mathbf{C}^{n\left(\lambda_{i}\right) \times n\left(\lambda_{i}\right)}$,

$\mathbf{J}_{i}^{(k)}\left(\lambda_{i}\right)=\left(\begin{array}{cccc}\lambda_{i} & 1 & & \\ & \ddots & \ddots & \\ & & \ddots & 1 \\ & & & \lambda_{i}\end{array}\right) \in \mathbf{C}^{n_{k}\left(\lambda_{i}\right) \times n_{k}\left(\lambda_{i}\right)}, \quad 1 \leq k \leq k_{i}, \quad 1 \leq i \leq r$,

$\sum_{k=1}^{k_{i}} n_{k}\left(\lambda_{i}\right)=n\left(\lambda_{i}\right), \quad 1 \leq i \leq r, \quad \sum_{i=1}^{r} n\left(\lambda_{i}\right)=n_{1}$, 
$\mathbf{N}=\operatorname{diag}\left(\mathbf{N}^{\left(l_{1}\right)}, \ldots, \mathbf{N}^{\left(l_{s}\right)}\right) \in \mathbf{C}^{n_{2} \times n_{2}}, \quad \mathbf{N}^{\left(l_{j}\right)}=\left(\begin{array}{cccc}0 & 1 & & \\ & \ddots & \ddots & \\ & & \ddots & 1 \\ & & & 0\end{array}\right) \in \mathbf{C}^{l_{j} \times l_{j}}, \quad 1 \leq j \leq s$, $\sum_{j=1}^{s} l_{j}=n_{2}, \quad n_{1}+n_{2}=n$.

Lemma 2. [14] Let $\mathbf{A}(p) \in \mathbf{C}^{n \times n}$ be an analytic matrix-valued function in some neighborhood $\aleph(0)$ of the origin, $\lambda_{1}$ is $r$ multiple defective eigenvalue of $\mathbf{A}(0)$, i.e., there exist invertible matrices $\mathbf{X}=\left(\mathbf{X}_{1}, \mathbf{X}_{2}\right) \in \mathbf{C}^{n \times n}, \mathbf{Y}=\left(\mathbf{Y}_{1}, \mathbf{Y}_{2}\right) \in \mathbf{C}^{n \times n}, \mathbf{X}_{\mathbf{1}}, \mathbf{Y}_{\mathbf{1}}, \in \mathbf{C}^{n \times r}$, such that:

$\mathbf{Y}^{\mathbf{H}} \mathbf{A}(0) \mathbf{X}=\left(\begin{array}{cc}\mathbf{A}_{1} & 0 \\ 0 & \mathbf{A}_{2}\end{array}\right), \quad \mathbf{Y}^{\mathbf{H}} \mathbf{X}=\mathbf{I}_{r}, \quad \lambda\left(\mathbf{A}_{1}\right)=\lambda_{1}, \lambda\left(\mathbf{A}_{1}\right) \cap \lambda\left(\mathbf{A}_{2}\right)=\emptyset$.

Then there exist a neighborhood of the origin $\aleph_{1}(0)$ and analytic matrix-valued functions $\mathbf{X}_{1}(p), \mathbf{Y}_{1}(p) \in \mathbf{C}_{r}^{n \times r}, \mathbf{A}_{1}(p) \in \mathbf{C}^{r \times r}$ on $\aleph_{1}(0)$ which satisfy:

1) $\mathbf{A}(p) \mathbf{X}_{1}(p)=\mathbf{X}_{1}(p) \mathbf{A}_{1}(p), \mathbf{Y}_{1}^{H}(p) \mathbf{A}(p)=\mathbf{A}_{1}(p) \mathbf{Y}_{1}^{H}(p), \mathbf{Y}_{1}^{H}(p) \mathbf{X}(p)=\mathbf{I}_{r}$.

2) $\mathbf{A}_{1}(0)=\mathbf{A}_{1}, \mathbf{X}_{1}(0)=\mathbf{X}_{1}, \mathbf{Y}_{1}(0)=\mathbf{Y}_{1}$.

3) Define $\lambda_{\text {aver }}=\operatorname{tr}\left(\mathbf{A}_{1}(p)\right) / r$, then $\lambda_{\text {aver }}$ is analytic on $\aleph_{1}(0)$ and:

$\frac{\partial \lambda_{\text {aver }}(0)}{\partial p_{i}}=\frac{1}{r} \operatorname{tr}\left(\mathbf{Y}_{1}^{H} \frac{\partial \mathbf{A}(0)}{\partial p_{i}} \mathbf{X}_{1}\right)$

4) $\frac{\partial \mathbf{X}_{1}(0)}{\partial p_{i}}=\mathbf{X}_{2} \Theta_{1}^{-1}\left(\mathbf{Y}_{2}^{H} \frac{\partial \mathbf{A}(0)}{\partial p_{i}} \mathbf{X}_{1}\right), \frac{\partial \mathbf{Y}_{1}^{H}(0)}{\partial p_{i}}=\Theta_{2}^{-1}\left(\mathbf{Y}_{1}^{H} \frac{\partial \mathbf{A}(0)}{\partial p_{i}} \mathbf{X}_{2}\right) \mathbf{Y}_{2}^{H}$, where:

$\Theta_{1}(\mathbf{V})=\mathbf{V} \mathbf{A}_{1}-\mathbf{A}_{2} \mathbf{V}, \Theta_{2}(\mathbf{V})=\mathbf{A}_{1} \mathbf{V}-\mathbf{V} \mathbf{A}_{2}$,

when $\lambda\left(\mathbf{A}_{1}\right) \cap \lambda\left(\mathbf{A}_{2}\right)=\emptyset, \Theta_{1}(\mathbf{V}), \Theta_{2}(\mathbf{V})$ is invertible.

Theorem 1. Let $p=\left(p_{1}, p_{2}, \ldots, p_{N}\right) \in C^{N}, \aleph(0)$ be a neighborhood of the origin of $\mathbf{C}^{N}, \mathbf{A}(p)$, $\mathbf{B}(p) \in \mathbf{C}^{n \times n}$ be analytic on $\aleph(0)$. Assume that $\mathbf{B}(p)$ is invertible on $\aleph(0)$ and $\lambda_{1}$ is a defective multiple eigenvalue of Eq. (1) at the origin with multiplicity $r(r>1)$. By Lemma 1 there exist invertible matrices $\mathbf{X}=\left(\mathbf{X}_{1}, \mathbf{X}_{2}\right) \in \mathbf{C}^{n \times n}, \mathbf{Y}=\left(\mathbf{Y}_{1}, \mathbf{Y}_{2}\right) \in \mathbf{C}^{n \times n}, \mathbf{X}_{\mathbf{1}}, \mathbf{Y}_{1}, \in \mathbf{C}^{n \times r}$, such that:

$\mathbf{Y}^{H} \mathbf{A}(0) \mathbf{X}=\left(\begin{array}{cc}\mathbf{A}_{1} & 0 \\ 0 & \mathbf{A}_{2}\end{array}\right), \quad \mathbf{Y}^{H} \mathbf{B}(0) \mathbf{X}=\mathbf{I}_{n}$

where $\mathbf{A}_{\mathbf{1}} \in \mathbf{C}^{r \times r}, \mathbf{A}_{2} \in \mathbf{C}^{(n-r) \times(n-r)}, \lambda\left(\mathbf{A}_{1}\right) \cap \lambda\left(\mathbf{A}_{2}\right)=\emptyset$. Then there exist a neighborhood of the origin $\aleph_{1}(0)$ and analytic matrix-valued functions $\mathbf{X}_{1}(p), \mathbf{Y}_{1}(p) \in \mathbf{C}_{r}^{n \times r}, \mathbf{A}_{1}(p) \in \mathbf{C}^{r \times r}$ on $\aleph_{1}(0)$ which satisfy:

1) $\mathbf{A}(p) \mathbf{X}_{1}(p)=\mathbf{B}(p) \mathbf{X}_{1}(p) \mathbf{A}_{1}(p), \mathbf{Y}_{1}^{H}(p) \mathbf{A}(p)=\mathbf{A}_{1}(p) \mathbf{Y}_{1}^{H}(p) \mathbf{B}(p)$

2) $\mathbf{A}_{1}(0)=\mathbf{A}_{1}, \mathbf{X}_{1}(0)=\mathbf{X}_{1}, \mathbf{Y}_{1}(0)=\mathbf{Y}_{1}$;

3) Define $\lambda_{\text {aver }}=\operatorname{tr}\left(\mathbf{A}_{1}(p)\right) / r$, then $\lambda_{\text {aver }}$ is analytic on $\aleph_{1}(0)$ and:

$$
\begin{aligned}
\frac{\partial \lambda_{\text {aver }}(0)}{\partial p_{i}} & =\frac{1}{r} \operatorname{tr}\left(\mathbf{Y}_{1}^{H} \frac{\partial \mathbf{A}(0)}{\partial p_{i}} \mathbf{X}_{1}-\mathbf{Y}_{1}^{H} \frac{\partial \mathbf{B}(0)}{\partial p_{i}} \mathbf{X}_{1} \mathbf{A}_{1}\right) \\
\text { 4) } \frac{\partial \mathbf{X}_{1}(0)}{\partial p_{i}} & =\mathbf{X}_{2} \Theta_{1}^{-1}\left(\mathbf{Y}_{2}^{H} \frac{\partial \mathbf{A}(0)}{\partial p_{i}} \mathbf{X}_{1}-\mathbf{Y}_{2}^{H} \frac{\partial \mathbf{B}(0)}{\partial p_{i}} \mathbf{X}_{1} \mathbf{A}_{1}\right) \\
\frac{\partial \mathbf{Y}_{1}^{H}(0)}{\partial p_{i}} & =\Theta_{2}^{-1}\left(\mathbf{Y}_{1}^{H} \frac{\partial \mathbf{A}(0)}{\partial p_{i}} X_{2}-\mathbf{Y}_{1}^{H} \frac{\partial \mathbf{B}(0)}{\partial p_{i}} \mathbf{X}_{2}\right) \mathbf{Y}_{2}^{H}-\mathbf{Y}_{1}^{H} \frac{\partial \mathbf{B}(0)}{\partial p_{i}} \mathbf{B}^{-1}(0)
\end{aligned}
$$


where $\Theta_{1}(\mathbf{V})=\mathbf{V} \mathbf{A}_{1}-\mathbf{A}_{2} \mathbf{V}, \Theta_{2}(\mathbf{V})=\mathbf{A}_{1} \mathbf{V}-\mathbf{V} \mathbf{A}_{2},\left(\right.$ when $\lambda\left(\mathbf{A}_{1}\right) \cap \lambda\left(\mathbf{A}_{2}\right)=\emptyset, \Theta_{1}(\mathbf{V}), \Theta_{2}(\mathbf{V})$ is invertible).

Proof. Let $\mathbf{C}(p)=\mathbf{B}^{-1}(p) \mathbf{A}(p)$, then Eq. (1) is equivalent to the following equation:

$\mathbf{C}(p) \mathbf{x}(p)=\lambda(p) \mathbf{x}(p), \quad \lambda(p) \in C, \quad \mathbf{x}(p) \in \mathbf{C}^{n}, \quad p \in \aleph(0)$.

Let $\widetilde{\mathbf{Y}}_{1}^{H}=\mathbf{Y}_{1}^{H} \mathbf{B}(0), \widetilde{\mathbf{Y}}_{2}^{H}=\mathbf{Y}_{2}^{H} \mathbf{B}(0), \widetilde{\mathbf{Y}}^{H}=\left(\widetilde{\mathbf{Y}}_{1}^{H}, \widetilde{\mathbf{Y}}_{2}^{H}\right)$, then $\widetilde{\mathbf{Y}}_{1}^{H}$ satisfies:

$\widetilde{\mathbf{Y}}_{1}^{H} \mathbf{C}(0) \mathbf{X}=\left(\begin{array}{cc}\mathbf{A}_{1} & 0 \\ 0 & \mathbf{A}_{2}\end{array}\right), \quad \widetilde{\mathbf{Y}}_{1}^{H} \mathbf{X}=\mathbf{I}_{n}$.

According to Lemma 2, there exist a neighborhood of the origin $\aleph_{1}(0)$ and analytic matrix-valued functions $\mathbf{X}_{1}(p), \mathbf{Y}_{1}(p) \in \mathbf{C}_{r}^{n \times r}, \mathbf{A}_{1}(p) \in \mathbf{C}^{r \times r}$ on $\aleph_{1}(0)$ which satisfy Eqs. (3)-(6):

$\mathbf{C}(p) \mathbf{X}_{1}(p)=\mathbf{X}_{1}(p) \mathbf{A}_{1}(p), \quad \widetilde{\mathbf{Y}}_{1}^{H}(p) \mathbf{C}(p)=\mathbf{A}_{1}(p) \widetilde{\mathbf{Y}}_{1}^{H}(p), \quad \widetilde{\mathbf{Y}}_{1}^{H}(p) \mathbf{X}(p)=\mathbf{I}_{r}$,

$\mathbf{A}_{1}(0)=\mathbf{A}_{1}, \quad \mathbf{X}_{1}(0)=\mathbf{X}_{1}, \quad \mathbf{Y}_{1}(0)=\mathbf{Y}_{1}$

$\lambda_{\text {aver }}=\operatorname{tr}\left(\mathbf{A}_{1}(p)\right) / r$ is analytic on $\aleph_{1}(0)$ and:

$\frac{\partial \lambda_{\text {aver }}(0)}{\partial p_{i}}=\frac{1}{r} \operatorname{tr}\left(\widetilde{\mathbf{Y}}_{1}^{H} \frac{\partial \mathbf{C}(0)}{\partial p_{i}} \mathbf{X}_{1}\right)$

$\frac{\partial \mathbf{X}_{1}(0)}{\partial p_{i}}=\mathbf{X}_{2} \Theta_{1}^{-1}\left(\widetilde{\mathbf{Y}}_{2}^{H} \frac{\partial \mathbf{C}(0)}{\partial p_{i}} \mathbf{X}_{1}\right), \quad \frac{\partial \mathbf{Y}_{1}^{H}(0)}{\partial p_{i}}=\Theta_{2}^{-1}\left(\widetilde{\mathbf{Y}}_{1}^{H} \frac{\partial \mathbf{C}(0)}{\partial p_{i}} \mathbf{X}_{2}\right) \widetilde{\mathbf{Y}}_{2}^{H}$,

where $\Theta_{1}(\mathbf{V})=\mathbf{V} \mathbf{A}_{1}-\mathbf{A}_{2} \mathbf{V}, \Theta_{2}(\mathbf{V})=\mathbf{A}_{1} \mathbf{V}-\mathbf{V} \mathbf{A}_{2}$, $\left(\right.$ when $\lambda\left(\mathbf{A}_{1}\right) \cap \lambda\left(\mathbf{A}_{2}\right)=\emptyset, \Theta_{1}(\mathbf{V}), \Theta_{2}(\mathbf{V})$ is invertible).

Let $\mathbf{Y}_{1}^{H}(p)=\widetilde{\mathbf{Y}}_{1}^{H}(p) \mathbf{B}^{-1}(P)$, from Eq. (3), we have:

$\mathbf{A}(p) \mathbf{X}_{1}(p)=\mathbf{B}(p) \mathbf{X}_{1}(p) \mathbf{A}_{1}(p), \quad \mathbf{Y}_{1}^{H}(p) \mathbf{A}(p)=\mathbf{A}_{1}(p) \mathbf{Y}_{1}^{H}(p) \mathbf{B}(p)$,

and $\mathbf{A}_{1}(0)=\mathbf{A}_{1}, \mathbf{X}_{1}(0)=\mathbf{X}_{1}, \mathbf{Y}_{1}(0)=\mathbf{Y}_{1}$, then we get Eqs. (1), (2) of the Theorem 1.

Because Eq. (1) is equivalent to the Eq. (2), the eigenvalues of Eq. (1) is same with Eq. (2), then the average of eigenvalues of Eq. (1) equals to the average of eigenvalues of Eq. (2). From Eq. (5), $\lambda_{\text {aver }}=\operatorname{tr}\left(\mathbf{A}_{1}(p)\right) / r$ is analytic on $\aleph_{1}(0)$ and:

$$
\begin{aligned}
& \frac{\partial \lambda_{\text {aver }}(0)}{\partial p_{i}}=\frac{1}{r} \operatorname{tr}\left(\widetilde{\mathbf{Y}}_{1}^{H} \frac{\partial \mathbf{C}(0)}{\partial p_{i}} \mathbf{X}_{1}\right)=\frac{1}{r} \operatorname{tr}\left\{\mathbf{Y}_{1}^{H} \mathbf{B}(0)\left[\mathbf{B}^{-1}(0)\left(\frac{\partial \mathbf{A}(0)}{\partial p_{i}}-\frac{\partial \mathbf{B}(0)}{\partial p_{i}} \mathbf{C}(0)\right)\right] \mathbf{X}_{1}\right\} \\
& \quad=\frac{1}{r} \operatorname{tr}\left[\mathbf{Y}_{1}^{H}\left(\frac{\partial \mathbf{A}(0)}{\partial p_{i}}-\frac{\partial \mathbf{B}(0)}{\partial p_{i}} \mathbf{B}^{-1}(0) \mathbf{A}(0)\right) \mathbf{X}_{1}\right]=\frac{1}{r} \operatorname{tr}\left(\mathbf{Y}_{1}^{H} \frac{\partial \mathbf{A}(0)}{\partial p_{i}} \mathbf{X}_{1}-\mathbf{Y}_{1}^{H} \frac{\partial \mathbf{B}(0)}{\partial p_{i}} \mathbf{X}_{1} \mathbf{A}_{1}\right) .
\end{aligned}
$$

From Eq. (6), we get the derivatives of right generalized eigenvectors matrices of Eq. (1):

$$
\begin{aligned}
& \frac{\partial \mathbf{X}_{1}(0)}{\partial p_{i}}=\mathbf{X}_{2} \Theta_{1}^{-1}\left(\widetilde{\mathbf{Y}}_{2}^{H} \frac{\partial \mathbf{C}(0)}{\partial p_{i}} \mathbf{X}_{1}\right)=\mathbf{X}_{2} \Theta_{1}^{-1}\left\{\mathbf{Y}_{2}^{H} \mathbf{B}(0)\left[\mathbf{B}^{-1}(0)\left(\frac{\partial \mathbf{A}(0)}{\partial p_{i}}-\frac{\partial \mathbf{B}(0)}{\partial p_{i}} \mathbf{C}(0)\right)\right] \mathbf{X}_{1}\right\} \\
& =\mathbf{X}_{2} \Theta_{1}^{-1}\left[\mathbf{Y}_{2}^{H}\left(\frac{\partial \mathbf{A}(0)}{\partial p_{i}}-\frac{\partial \mathbf{B}(0)}{\partial p_{i}} \mathbf{B}^{-1}(0) \mathbf{A}(0)\right) \mathbf{X}_{1}\right] \\
& =\mathbf{X}_{2} \Theta_{1}^{-1}\left(\mathbf{Y}_{2}^{H} \frac{\partial \mathbf{A}(0)}{\partial p_{i}} \mathbf{X}_{1}-\mathbf{Y}_{2}^{H} \frac{\partial \mathbf{B}(0)}{\partial p_{i}} \mathbf{X}_{1} \mathbf{A}_{1}\right) .
\end{aligned}
$$


According to:

$$
\begin{aligned}
& \frac{\partial \widetilde{\mathbf{Y}}_{1}^{H}(0)}{\partial p_{i}}=\Theta_{2}^{-1}\left(\widetilde{\mathbf{Y}}_{1}^{H} \frac{\partial \mathbf{C}(0)}{\partial p_{i}} \mathbf{X}_{2}\right) \widetilde{\mathbf{Y}}_{2}^{H} \\
& \quad=\Theta_{2}^{-1}\left\{\mathbf{Y}_{1}^{H} \mathbf{B}(0)\left[\mathbf{B}^{-1}(0)\left(\frac{\partial \mathbf{A}(0)}{\partial p_{i}}-\frac{\partial \mathbf{B}(0)}{\partial p_{i}} \mathbf{C}(0)\right)\right] \mathbf{X}_{2}\right\} \mathbf{Y}_{2}^{H} \mathbf{B}(0) \\
& \quad=\Theta_{2}^{-1}\left(\mathbf{Y}_{1}^{H} \frac{\partial \mathbf{A}(0)}{\partial p_{i}} \mathbf{X}_{2}-\mathbf{Y}_{1}^{H} \frac{\partial \mathbf{B}(0)}{\partial p_{i}} \mathbf{X}_{2} \mathbf{A}_{2}\right) \mathbf{Y}_{2}^{H} \mathbf{B}(0),
\end{aligned}
$$

and $\widetilde{\mathbf{Y}}_{1}^{H}(p)=\mathbf{Y}_{1}^{H}(p) \mathbf{B}(P)$, we get the derivatives of left generalized eigenvectors matrices of Eq. (1):

$$
\begin{aligned}
& \frac{\partial \mathbf{Y}_{1}^{H}(0)}{\partial p_{i}}=\frac{\widetilde{\mathbf{Y}}_{1}^{H}(0)}{\partial p_{i}} \mathbf{B}^{-1}(0)-\mathbf{Y}_{1}^{H} \frac{\partial \mathbf{B}(0)}{\partial p_{i}} \mathbf{B}^{-1}(0) \\
& \quad=\Theta_{2}^{-1}\left(\mathbf{Y}_{1}^{H} \frac{\partial \mathbf{A}(0)}{\partial p_{i}} \mathbf{X}_{2}-\mathbf{Y}_{1}^{H} \frac{\partial \mathbf{B}(0)}{\partial p_{i}} \mathbf{X}_{2} \mathbf{A}_{2}\right) \mathbf{Y}_{2}^{H}-\mathbf{Y}_{1}^{H} \frac{\partial \mathbf{B}(0)}{\partial p_{i}} \mathbf{B}^{-1}(0)
\end{aligned}
$$

So the proof of the Theorem 1 is completed.

\section{Sensitivity of defective eigenpairs of quadratic eigenvalue problem}

In this section we study sensitivity of the following quadratic eigenvalue problem:

$$
\left(\lambda^{2}(p) \mathbf{M}(p)+\lambda(p) \mathbf{C}(p)+\mathbf{K}(p)\right) \mathbf{u}(p)=0, \lambda(p) \in \mathbf{C}, \mathbf{x}(p) \in \mathbf{C}^{n}, \quad p \in \aleph(0),
$$

where $p=\left(p_{1}, p_{2}, \ldots, p_{N}\right) \in \mathbf{C}^{N}, \mathbf{M}(p), \mathbf{K}(p), \mathbf{C}(p) \in \mathbf{C}^{n \times n}$ are analytic matrix-valued functions in some neighborhood $\mathrm{N}\left(p^{*}\right)$ of the point $p^{*}$ and $\mathbf{M}(p)$ is invertible. Without loss of generality we may assume that the point $p^{*}$ is the origin of $\mathbf{C}^{N}$.

Lemma 3 [38]. Suppose $\mathbf{M}, \mathbf{C}, \mathbf{K} \in \mathbf{C}^{n \times n}$ and $\mathbf{M}$ is invertible, let:

$$
\mathbf{A}=\left(\begin{array}{cc}
-\mathbf{K} & \mathbf{0} \\
\mathbf{0} & \mathbf{M}
\end{array}\right), \quad \mathbf{B}=\left(\begin{array}{ll}
\mathbf{C} & \mathbf{M} \\
\mathbf{M} & \mathbf{0}
\end{array}\right)
$$

Then, we have:

1) Quadratic eigenvalue problem Eq. (7) is equivalent to generalized eigenvalue problem $\mathbf{A x}=\lambda \mathbf{B x}$, where $\mathbf{x}^{H}=\left[\mathbf{u}^{H}, \lambda \mathbf{u}^{H}\right]$.

2) Suppose $\lambda$ is the eigenvalue of Eq. (7), the columns of $\mathbf{U}, \mathbf{V} \in \mathbf{C}^{n \times r}$ are the corresponding right eigenvectors and left eigenvectors of $\lambda$, let $\mathbf{X}^{H}=\left[\mathbf{U}^{H}, \lambda \mathbf{U}^{H}\right], \mathbf{Y}^{H}=\left[\mathbf{V}^{H}, \lambda \mathbf{V}^{H}\right]$, then:

$\mathbf{A X}=\lambda \mathbf{B X}, \mathbf{Y}^{H} \mathbf{A}=\lambda \mathbf{Y}^{H} \mathbf{B}, \quad \mathbf{Y}^{H} \mathbf{B X}=\mathbf{V}^{H}(2 \lambda \mathbf{M}+\mathbf{C}) \mathbf{U}$.

3) If $\mathbf{U}_{1} \in \mathbf{C}_{r}^{n \times r}, \mathbf{D}_{1} \in \mathbf{C}^{r \times r}$ satisfy $\mathbf{M U}_{1} \mathbf{D}_{1}^{2}+\mathbf{C U}_{1} \mathbf{D}_{1}+\mathbf{K} \mathbf{U}_{1}=\mathbf{0}$, then $\lambda\left(\mathbf{D}_{1}\right) \subset \lambda(\mathbf{M}, \mathbf{C}, \mathbf{K})$.

Theorem 2. Suppose $\mathbf{M}, \mathbf{C}, \mathbf{K} \in \mathbf{C}^{n \times n}$ and $\mathbf{M}$ is invertible. If $\lambda_{1}$ is a defective multiple eigenvalues of Eq. (7) with multiplicity $r$. Then there exist:

$\mathbf{U}=\left(\mathbf{U}_{1}, \mathbf{U}_{2}\right) \in \mathbf{C}^{n \times 2 n}, \quad \mathbf{V}=\left(\mathbf{V}_{1}, \mathbf{V}_{2}\right) \in \mathbf{C}^{n \times 2 n}, \quad \mathbf{U}_{1}, \mathbf{V}_{1} \in \mathbf{C}_{r}^{n \times r}$, $\mathbf{A}_{1} \in \mathbf{C}^{r \times r}, \quad \mathbf{A}_{2} \in \mathbf{C}^{(2 n-r) \times(2 n-r)}$,

such that $\mathbf{U}_{1}, \mathbf{V}_{1}$ are right and left eigenvector matrices corresponding to eigenvalues matrix $A_{1}$ respectively, $\mathbf{U}_{2}, \mathbf{V}_{2}$ are right and left eigenvector matrices corresponding to eigenvalues matrix $\mathbf{A}_{2}$ respectively. Moreover: 
$\mathbf{V}_{1}^{H} \mathbf{C U}_{1}+\mathbf{A}_{1} \mathbf{V}_{1}^{H} \mathbf{M} \mathbf{U}_{1}+\mathbf{V}_{1}^{H} \mathbf{M} \mathbf{U}_{1} \mathbf{A}_{1}=\mathbf{I}_{r}$

$\mathbf{V}_{2}^{H} \mathbf{C} \mathbf{U}_{2}+\mathbf{A}_{2} \mathbf{V}_{2}^{H} \mathbf{M} \mathbf{U}_{2}+\mathbf{V}_{2}^{H} \mathbf{M} \mathbf{U}_{2} \mathbf{A}_{2}=\mathbf{I}_{2 n-r}$,

$\lambda\left(\mathbf{A}_{1}\right)=\lambda_{1}, \quad \lambda_{1} \notin \lambda\left(\mathbf{A}_{2}\right)$.

Proof. Since $\lambda_{1}$ is a defective multiple eigenvalues of Eq. (7) with multiplicity $r$, from Lemma $3, \lambda_{1}$ is also a defective multiple eigenvalues of $\mathbf{A x}=\lambda \mathbf{B} \mathbf{x}$, where:

$\mathbf{A}=\left(\begin{array}{cc}-\mathbf{K} & \mathbf{0} \\ \mathbf{0} & \mathbf{M}\end{array}\right), \quad \mathbf{B}=\left(\begin{array}{cc}\mathbf{C} & \mathbf{M} \\ \mathbf{M} & \mathbf{0}\end{array}\right)$

By Lemma 1, there exists $\mathbf{X}=\left(\mathbf{X}_{1}, \mathbf{X}_{2}\right), \mathbf{Y}=\left(\mathbf{Y}_{1}, \mathbf{Y}_{2}\right), \mathbf{X}_{1}, \mathbf{Y}_{1} \in C^{2 n \times r}$ such that:

$\mathbf{Y}^{H} \mathbf{A X}=\left(\begin{array}{cc}\mathbf{A}_{1} & \mathbf{0} \\ \mathbf{0} & \mathbf{A}_{2}\end{array}\right), \quad \mathbf{Y}^{H} \mathbf{B X}=\mathbf{I}_{2 n}, \quad \lambda_{1} \notin \lambda\left(\mathbf{A}_{2}\right)$.

From Eq. (8) we get:

$\mathbf{A X}_{1}=\mathbf{B} \mathbf{X}_{1} \mathbf{A}_{1}, \quad \mathbf{Y}_{1}^{H} \mathbf{A}=\mathbf{A}_{1} \mathbf{Y}_{1}^{H} \mathbf{B}$

$\mathbf{A X}_{2}=\mathbf{B} \mathbf{X}_{2} \mathbf{A}_{2}, \quad \mathbf{Y}_{2}^{H} \mathbf{A}=\mathbf{A}_{2} \mathbf{Y}_{2}^{H} \mathbf{B}$,

$\mathbf{Y}_{1}^{H} \mathbf{B} \mathbf{X}_{1}=\mathbf{I}_{r}, \quad \mathbf{Y}_{2}^{H} \mathbf{B} \mathbf{X}_{2}=\mathbf{I}_{2 n-r}$.

Let $\mathbf{U}_{i}=\left[\mathbf{I}_{n}, \mathbf{0}\right] \mathbf{X}_{i}, \mathbf{V}_{i}=\left[\mathbf{I}_{n}, \mathbf{0}\right] \mathbf{Y}_{i}(i=1,2)$, from Eq. (9) we have:

$\mathbf{X}_{1}^{H}=\left[\mathbf{U}_{1}^{H}, \mathbf{A}_{1}^{H} \mathbf{U}_{1}^{H}\right], \quad \mathbf{Y}_{1}^{H}=\left[\mathbf{V}_{1}^{H}, \mathbf{A}_{1} \mathbf{V}_{1}^{H}\right]$,

$\mathbf{M U}_{1} \mathbf{A}_{1}^{2}+\mathbf{C U}_{1} \mathbf{A}_{1}+\mathbf{K} \mathbf{U}_{1}=\mathbf{0}, \quad \mathbf{A}_{1}^{2} \mathbf{V}_{1}^{H} \mathbf{M}+\mathbf{A}_{1} \mathbf{V}_{1}^{H} \mathbf{C}+\mathbf{V}_{1}^{H} \mathbf{K}=\mathbf{0}$.

From Eq. (10) we get:

$\mathbf{X}_{2}^{H}=\left[\mathbf{U}_{2}^{H}, \mathbf{A}_{2}^{H} \mathbf{U}_{2}^{H}\right], \quad \mathbf{Y}_{2}^{H}=\left[\mathbf{V}_{2}^{H}, \mathbf{A}_{2} \mathbf{V}_{2}^{H}\right]$,

$\mathbf{M U}_{2} \mathbf{A}_{2}^{2}+\mathbf{C U}_{2} \mathbf{A}_{2}+\mathbf{K} \mathbf{U}_{2}=\mathbf{0}, \quad \mathbf{A}_{2}^{2} \mathbf{V}_{2}^{H} \mathbf{M}+\mathbf{A}_{2} \mathbf{V}_{2}^{H} \mathbf{C}+\mathbf{V}_{2}^{H} \mathbf{K}=\mathbf{0}$.

Thus $\mathbf{U}_{\mathbf{1}}$ and $\mathbf{V}_{\mathbf{1}}$ are right and left eigenvector matrices corresponding to eigenvalues matrix $\mathbf{A}_{\mathbf{1}}$ respectively, $\mathbf{U}_{\mathbf{2}}$ and $\mathbf{V}_{\mathbf{2}}$ are right and left eigenvector matrices corresponding to eigenvalues matrix $\mathbf{A}_{2}$ respectively. From Eqs. (11), (12) and (14) we get:

$\mathbf{V}_{1}^{H} \mathbf{C U}_{1}+\mathbf{A}_{1} \mathbf{V}_{1}^{H} \mathbf{M U}_{1}+\mathbf{V}_{1}^{H} \mathbf{M} \mathbf{U}_{1} \mathbf{A}_{1}=\mathbf{I}_{r}$

$\mathbf{V}_{2}^{H} \mathbf{C U}_{2}+\mathbf{A}_{2} \mathbf{V}_{2}^{H} \mathbf{M} \mathbf{U}_{2}+\mathbf{V}_{2}^{H} \mathbf{M U}_{2} \mathbf{A}_{2}=\mathbf{I}_{2 n-r}$.

So the proof of the theorem is completed.

Theorem 3. Let $p=\left(p_{1}, p_{2}, \ldots, p_{N}\right) \in \mathbf{C}^{N}, \aleph(0)$ be a neighborhood of the origin of $\mathbf{C}^{N}, \mathbf{M}(p)$, $\mathbf{C}(p), \mathbf{K}(p) \in C^{n \times n}$ be analytic on $\aleph(0)$. Assume that $\mathbf{M}(p)$ is invertible on $\aleph(0)$ and $\lambda_{1}$ is a defective multiple eigenvalue of Eq. (7) at the origin with multiplicity $r(r>1)$, i.e., there exist invertible matrices $\mathbf{U}=\left(\mathbf{U}_{1}, \mathbf{U}_{2}\right) \in \mathbf{C}^{n \times 2 n}, \mathbf{V}=\left(\mathbf{V}_{1}, \mathbf{V}_{2}\right) \in \mathbf{C}^{n \times 2 n}, \mathbf{U}_{1}, \mathbf{V}_{1} \in \mathbf{C}_{r}^{n \times r}, \mathbf{A}_{1} \in \mathbf{C}^{r \times r}$, $\mathbf{A}_{2} \in \mathbf{C}^{(2 n-r) \times(2 n-r)}$, such that $\mathbf{U}_{1}, \mathbf{V}_{1}$ are right and left eigenvector matrices corresponding to eigenvalues matrix $\mathbf{A}_{1}$ respectively, $\mathbf{U}_{2}, \mathbf{V}_{2}$ are right and left eigenvector matrices corresponding to eigenvalues matrix $\mathbf{A}_{2}$ respectively. Moreover:

$\mathbf{V}_{1}^{H} \mathbf{C U}_{1}+\mathbf{A}_{1} \mathbf{V}_{1}^{H} \mathbf{M} \mathbf{U}_{1}+\mathbf{V}_{1}^{H} \mathbf{M} \mathbf{U}_{1} \mathbf{A}_{1}=\mathbf{I}_{r}$

$\mathbf{V}_{2}^{H} \mathbf{C U}_{2}+\mathbf{A}_{2} \mathbf{V}_{2}^{H} \mathbf{M} \mathbf{U}_{2}+\mathbf{V}_{2}^{H} \mathbf{M} \mathbf{U}_{2} \mathbf{A}_{2}=\mathbf{I}_{2 n-r}$,

$\lambda\left(\mathbf{A}_{1}\right)=\lambda_{1}, \quad \lambda_{1} \notin \lambda\left(\mathbf{A}_{2}\right)$.

Then there exist a neighborhood of the origin $\aleph_{1}(0)$ and analytic matrix-valued functions $\mathbf{U}_{1}(p), \mathbf{V}_{1}(p) \in \mathbf{C}_{r}^{n \times r}, \mathbf{A}_{1}(p) \in \mathbf{C}^{r \times r}$ on $\aleph_{1}(0)$ which satisfy: 
1) $\mathbf{M}(p) \Upsilon_{1}(p) \mathbf{A}_{1}^{2}(p)+\mathbf{C}(p) \mathbf{U}_{1}(p) \mathbf{A}_{1}(p)+\mathbf{K}(p) \mathbf{U}_{1}(p)=\mathbf{0}$

$\mathbf{A}_{1}^{2}(p) \mathbf{V}_{1}^{H}(p) \mathbf{M}(p)+\mathbf{A}_{1}(p) \mathbf{V}_{1}^{H}(p) \mathbf{C}(p)+\mathbf{V}_{1}^{H}(p) \mathbf{K}(p)=\mathbf{0}$.

2) $\mathbf{A}_{1}(0)=\mathbf{A}_{1}, \mathbf{U}_{1}(0)=\mathbf{U}_{1}, \mathbf{V}_{1}(0)=\mathbf{V}_{1}$.

3) The average of the eigenvalues $\lambda_{\text {aver }}=\operatorname{tr}\left(\mathbf{A}_{1}(p)\right) / r$ is analytic on $\aleph_{1}(0)$ and:

$$
\begin{aligned}
\frac{\partial \lambda_{\text {aver }}(0)}{\partial p_{i}} & =\frac{1}{r} \operatorname{tr}\left[-\mathbf{V}_{1}^{H}\left(\frac{\partial \mathbf{M}(0)}{\partial p_{i}} U_{1} A_{1}^{2}+\frac{\partial \mathbf{C}(0)}{\partial p_{i}} U_{1} A_{1}+\frac{\partial \mathbf{K}(0)}{\partial p_{i}} \mathbf{U}_{1}\right)\right] . \\
\text { 4) } \frac{\partial \mathbf{U}_{1}(0)}{\partial p_{i}} & =\mathbf{U}_{2} \Theta_{1}^{-1}\left[-\mathbf{V}_{2}^{H}\left(\frac{\partial \mathbf{M}(0)}{\partial p_{i}} \mathbf{U}_{1} A_{1}^{2}+\frac{\partial \mathbf{C}(0)}{\partial p_{i}} U_{1} A_{1}+\frac{\partial \mathbf{K}(0)}{\partial p_{i}} \mathbf{U}_{1}\right)\right], \\
\frac{\partial \mathbf{V}_{1}^{H}(0)}{\partial p_{i}} & =\Theta_{2}^{-1}\left[-\mathbf{V}_{1}^{H}\left(\frac{\partial \mathbf{M}(0)}{\partial p_{i}} \mathbf{U}_{2} \mathbf{A}_{2}^{2}+\frac{\partial \mathbf{C}(0)}{\partial p_{i}} \mathbf{U}_{2} \mathbf{A}_{2}+\frac{\partial \mathbf{K}(0)}{\partial p_{i}} \mathbf{U}_{2}\right)\right] \mathbf{V}_{2}^{H}-V_{1}^{H} \frac{\partial \mathbf{M}(0)}{\partial p_{i}} \mathbf{M}^{-1}(0),
\end{aligned}
$$

where $\Theta_{1}(\mathbf{V})=\mathbf{V} \mathbf{A}_{1}-\mathbf{A}_{2} \mathbf{V}, \Theta_{2}(\mathbf{V})=\mathbf{A}_{1} \mathbf{V}-\mathbf{V} \mathbf{A}_{2},\left(\right.$ when $\lambda\left(\mathbf{A}_{1}\right) \cap \lambda\left(\mathbf{A}_{2}\right)=\emptyset, \Theta_{1}, \Theta_{2}$ is invertible).

Proof. Let:

$$
\begin{aligned}
& \mathbf{A}(p)=\left(\begin{array}{cc}
-\mathbf{K}(p) & 0 \\
0 & \mathbf{M}(p)
\end{array}\right), \quad \mathbf{B}(p)=\left(\begin{array}{cc}
\mathbf{C}(p) & \mathbf{M}(p) \\
\mathbf{M}(p) & \mathbf{0}
\end{array}\right), \quad \mathbf{x}(p)=\left(\begin{array}{c}
\mathbf{u}(p) \\
\lambda(p) \mathbf{u}(p)
\end{array}\right), \\
& \mathbf{X}=\left(\mathbf{X}_{1}, \mathbf{X}_{2}\right), \quad \mathbf{X}_{1}^{H}=\left[\mathbf{U}_{1}^{H}, \mathbf{A}_{1}^{H} \mathbf{U}_{1}^{H}\right], \quad \mathbf{X}_{2}^{H}=\left[\mathbf{U}_{2}^{H}, \mathbf{A}_{2}^{H} \mathbf{U}_{2}^{H}\right], \\
& \mathbf{Y}=\left(\mathbf{Y}_{1}, \mathbf{Y}_{2}\right), \quad \mathbf{Y}_{1}^{H}=\left[\mathbf{V}_{1}^{H}, \mathbf{A}_{1} \mathbf{V}_{1}^{H}\right], \quad \mathbf{Y}_{2}^{H}=\left[\mathbf{V}_{2}^{H}, \mathbf{A}_{2} \mathbf{V}_{2}^{H}\right] .
\end{aligned}
$$

From Lemma 3 and the proof of Theorem 2, Eq. (7) is equivalent to generalized eigenvalue problem $\mathbf{A}(p) \mathbf{x}(p)=\lambda(p) \mathbf{B}(p) \mathbf{x}(p), \mathbf{B}(p)$ is invertible, $\lambda_{1}$ is a defective multiple eigenvalue of $\{\mathbf{A}(0), \mathbf{B}(0)\}$ at the origin with multiplicity $r$ and:

$\mathbf{Y}^{H} \mathbf{A}(0) \mathbf{X}=\left(\begin{array}{cc}\mathbf{A}_{1} & 0 \\ 0 & \mathbf{A}_{2}\end{array}\right), \quad \mathbf{Y}^{H} \mathbf{B X}=\mathbf{I}_{2 n}, \quad \lambda_{1} \notin \lambda\left(\mathbf{A}_{2}\right)$.

According to Theorem 1, Then there exist a neighborhood of the origin $\aleph_{1}(0)$ and analytic matrix-valued functions $\mathbf{X}_{1}(p), \quad \mathbf{Y}_{1}(p) \in \mathbf{C}_{r}^{2 n \times r}, \quad \mathbf{A}_{1}(p) \in \mathbf{C}^{r \times r}$ on $\aleph_{1}(0)$ which satisfy Eqs. (18-22):

$$
\begin{aligned}
& \mathbf{A}(p) \mathbf{X}_{1}(p)=\mathbf{B}(p) \mathbf{X}_{1}(p) \mathbf{A}_{1}(p), \quad \mathbf{Y}_{1}^{H}(p) \mathbf{A}(p)=\mathbf{A}_{1}(p) \mathbf{Y}_{1}^{H}(p) \mathbf{B}(p) \\
& \mathbf{A}_{1}(0)=\mathbf{A}_{1}, \quad \mathbf{X}_{1}(0)=\mathbf{X}_{1}, \quad \mathbf{Y}_{1}(0)=\mathbf{Y}_{1} .
\end{aligned}
$$

The average of the eigenvalues $\lambda_{\text {aver }}=\operatorname{tr}\left(\mathbf{A}_{1}(p)\right) / r$ is analytic on $\aleph_{1}(0)$ and:

$$
\begin{aligned}
& \frac{\partial \lambda_{\text {aver }}(0)}{\partial p_{i}}=\frac{1}{r} \operatorname{tr}\left(\mathbf{Y}_{1}^{H} \frac{\partial \mathbf{A}(0)}{\partial p_{i}} \mathbf{X}_{1}-\mathbf{Y}_{1}^{H} \frac{\partial \mathbf{B}(0)}{\partial p_{i}} \mathbf{X}_{1} \mathbf{A}_{1}\right) \\
& \frac{\partial \mathbf{X}_{1}(0)}{\partial p_{i}}=\mathbf{X}_{2} \Theta_{1}^{-1}\left(\mathbf{Y}_{2}^{H} \frac{\partial \mathbf{A}(0)}{\partial p_{i}} \mathbf{X}_{1}-\mathbf{Y}_{2}^{H} \frac{\partial \mathbf{B}(0)}{\partial p_{i}} \mathbf{X}_{1} \mathbf{A}_{1}\right) \\
& \frac{\partial \mathbf{Y}_{1}^{H}(0)}{\partial p_{i}}=\Theta_{2}^{-1}\left(\mathbf{Y}_{1}^{H} \frac{\partial \mathbf{A}(0)}{\partial p_{i}} X_{2}-\mathbf{Y}_{1}^{H} \frac{\partial \mathbf{B}(0)}{\partial p_{i}} \mathbf{X}_{2}\right) \mathbf{Y}_{2}^{H}-\mathbf{Y}_{1}^{H} \frac{\partial \mathbf{B}(0)}{\partial p_{i}} \mathbf{B}^{-1}(0)
\end{aligned}
$$

where $\Theta_{1}(\mathbf{V})=\mathbf{V} \mathbf{A}_{1}-\mathbf{A}_{2} \mathbf{V}, \Theta_{2}(\mathbf{V})=\mathbf{A}_{1} \mathbf{V}-\mathbf{V} \mathbf{A}_{2}$, $\left(\right.$ when $\lambda\left(\mathbf{A}_{1}\right) \cap \lambda\left(\mathbf{A}_{2}\right)=\emptyset, \Theta_{1}(\mathbf{V}), \Theta_{2}(\mathbf{V})$ is invertible).

Let $\mathbf{U}_{i}(p)=\left[\mathbf{I}_{n}, \mathbf{0}\right] \mathbf{X}_{i}(p), \mathbf{V}_{i}(p)=\left[\mathbf{I}_{n}, \mathbf{0}\right] \mathbf{Y}_{i}(p),(i=1,2)$, then $\mathbf{U}_{1}(p), \mathbf{V}_{1}(p)$ is analytic on $\aleph_{1}(0)$. From Eq. (18) we get:

$\mathbf{M}(p) \mathbf{U}_{1}(p) \mathbf{A}_{1}^{2}(p)+\mathbf{C}(p) \mathbf{U}_{1}(p) \mathbf{A}_{1}(p)+\mathbf{K}(p) \mathbf{U}_{1}(p)=\mathbf{0}$,

$\mathbf{A}_{1}^{2}(p) \mathbf{V}_{1}^{H}(p) \mathbf{M}(p)+\mathbf{A}_{1}(p) \mathbf{V}_{1}^{H}(p) \mathbf{C}(p)+\mathbf{V}_{1}^{H}(p) \mathbf{K}(p)=\mathbf{0}$. 
Thus $\mathbf{U}_{1}(p), \mathbf{V}_{1}(p)$ are the right and left eigenvector matrices corresponding to eigenvalues matrix $\mathbf{A}_{1}(p)$ of the Eq. (7) respectively. By Eq. (19), $\mathbf{U}_{1}(0)=\mathbf{U}_{1}, \mathbf{V}_{1}(0)=\mathbf{V}_{1}$, so we get Eqs. (1), (2) of this theorem. By Eq. (20), the average of the eigenvalues $\lambda_{\text {aver }}=\operatorname{tr}\left(\mathbf{A}_{1}(p)\right) / r$ is analytic on $\aleph_{1}(0)$ and:

$$
\frac{\partial \lambda_{\text {aver }}(0)}{\partial p_{i}}=\frac{1}{r} \operatorname{tr}\left(\mathbf{Y}_{1}^{H} \frac{\partial \mathbf{A}(0)}{\partial p_{i}} \mathbf{X}_{1}-\mathbf{Y}_{1}^{H} \frac{\partial \mathbf{B}(0)}{\partial p_{i}} \mathbf{X}_{1} \mathbf{A}_{1}\right)
$$

Since:

$$
\begin{aligned}
& \mathbf{Y}_{1}^{H} \frac{\partial \mathbf{A}(0)}{\partial p_{i}} \mathbf{X}_{1}-\mathbf{Y}_{1}^{H} \frac{\partial \mathbf{B}(0)}{\partial p_{i}} \mathbf{X}_{1} \mathbf{A}_{1}=\left(\mathbf{V}_{1}, \mathbf{A}_{1} \mathbf{V}_{1}^{H}\right)\left(\begin{array}{cc}
-\frac{\partial \mathbf{K}(0)}{\partial p_{i}} & \\
\frac{\partial \mathbf{M}(0)}{\partial p_{i}}
\end{array}\right)\left(\begin{array}{c}
\mathbf{U}_{1} \\
\mathbf{U}_{1} \mathbf{A}_{1}
\end{array}\right) \\
& -\left(\mathbf{V}_{1}, \mathbf{A}_{1} \mathbf{V}_{1}^{H}\right)\left(\begin{array}{cc}
\frac{\partial \mathbf{C}(0)}{\partial p_{i}} & \frac{\partial \mathbf{M}(0)}{\partial p_{i}} \\
\frac{\partial \mathbf{M}(0)}{\partial p_{i}} & \mathbf{0}
\end{array}\right)\left(\begin{array}{c}
\mathbf{U}_{1} \\
\mathbf{U}_{1} \mathbf{A}_{1}
\end{array}\right) \mathbf{A}_{1} \\
& =-\mathbf{V}_{1}^{H}\left(\frac{\partial \mathbf{M}(0)}{\partial p_{i}} \mathbf{U}_{1} \mathbf{A}_{1}^{2}+\frac{\partial \mathbf{C}(0)}{\partial p_{i}} \mathbf{U}_{1} \mathbf{A}_{1}+\frac{\partial \mathbf{K}(0)}{\partial p_{i}} \mathbf{U}_{1}\right) .
\end{aligned}
$$

Then:

$\frac{\partial \lambda_{\text {aver }}(0)}{\partial p_{i}}=\frac{1}{r} \operatorname{tr}\left[-\mathbf{V}_{1}^{H}\left(\frac{\partial \mathbf{M}(0)}{\partial p_{i}} \mathbf{U}_{1} \mathbf{A}_{1}^{2}+\frac{\partial \mathbf{C}(0)}{\partial p_{i}} \mathbf{U}_{1} \mathbf{A}_{1}+\frac{\partial \mathbf{K}(0)}{\partial p_{i}} \mathbf{U}_{1}\right)\right]$

so we get Eq. (3) of the theorem. According to Eq. (21):

$\frac{\partial \mathbf{U}_{1}(0)}{\partial p_{i}}=\mathbf{U}_{2} \Theta_{1}^{-1}\left(\mathbf{Y}_{2}^{H} \frac{\partial \mathbf{A}(0)}{\partial p_{i}} \mathbf{X}_{1}-\mathbf{Y}_{2}^{H} \frac{\partial \mathbf{B}(0)}{\partial p_{i}} \mathbf{X}_{1} \mathbf{A}_{1}\right)$

Since:

$$
\begin{aligned}
& \mathbf{Y}_{2}^{H} \frac{\partial \mathbf{A}(0)}{\partial p_{i}} \mathbf{X}_{1}-\mathbf{Y}_{2}^{H} \frac{\partial \mathbf{B}(0)}{\partial \mathbf{p}_{i}} \mathbf{X}_{1} \mathbf{A}_{1}=\left(\mathbf{V}_{2}^{H}, \mathbf{A}_{2} \mathbf{V}_{2}^{H}\right)\left(\begin{array}{cc}
-\frac{\partial \mathbf{K}(0)}{\partial p_{i}} & \\
\frac{\partial \mathbf{M}(0)}{\partial p_{i}}
\end{array}\right)\left(\begin{array}{c}
\mathbf{U}_{1} \\
\mathbf{U}_{1} \mathbf{A}_{1}
\end{array}\right) \\
& -\left(\mathbf{V}_{2}^{H}, \mathbf{A}_{2} \mathbf{V}_{2}^{H}\right)\left(\begin{array}{cc}
\frac{\partial \mathbf{C}(0)}{\partial p_{i}} & \frac{\partial \mathbf{M}(0)}{\partial p_{i}} \\
\frac{\partial \mathbf{M}(0)}{\partial p_{i}} & \mathbf{0}
\end{array}\right)\left(\begin{array}{l}
\mathbf{U}_{1} \\
\mathbf{U}_{1} \mathbf{A}_{1}
\end{array}\right) \mathbf{A}_{1} \\
& \quad=-\mathbf{V}_{2}^{H}\left(\frac{\partial \mathbf{M}(0)}{\partial p_{i}} U_{1} A_{1}^{2}+\frac{\partial \mathbf{C}(0)}{\partial p_{i}} U_{1} A_{1}+\frac{\partial \mathbf{K}(0)}{\partial p_{i}} \mathbf{U}_{1}\right) .
\end{aligned}
$$

Then: 
$\frac{\partial \mathbf{U}_{1}(0)}{\partial p_{i}}=\mathbf{U}_{2} \Theta_{1}^{-1}\left[-\mathbf{V}_{2}^{H}\left(\frac{\partial \mathbf{M}(0)}{\partial p_{i}} U_{1} A_{1}^{2}+\frac{\partial \mathbf{C}(0)}{\partial p_{i}} U_{1} A_{1}+\frac{\partial \mathbf{K}(0)}{\partial p_{i}} \mathbf{U}_{1}\right)\right]$

Utilizing Eq. (22) and the following equation:

$$
\begin{aligned}
& \mathbf{Y}_{1}^{H} \frac{\partial \mathbf{A}(0)}{\partial p_{i}} \mathbf{X}_{2}-\mathbf{Y}_{1}^{H} \frac{\partial \mathbf{B}(0)}{\partial p_{i}} \mathbf{X}_{2}=\left(\mathbf{V}_{1}^{H}, \mathbf{A}_{1} \mathbf{V}_{1}^{H}\right)\left(\begin{array}{ll}
-\frac{\partial \mathbf{K}(0)}{\partial p_{i}} & \\
\frac{\partial \mathbf{M}(0)}{\partial p_{i}}
\end{array}\right)\left(\begin{array}{c}
\mathbf{U}_{2} \\
\mathbf{U}_{2} \mathbf{A}_{2}
\end{array}\right) \\
& -\left(\begin{array}{ll}
\frac{\partial \mathbf{C}(0)}{\partial p_{i}} & \frac{\partial \mathbf{M}(0)}{\partial p_{i}} \\
\frac{\partial \mathbf{M}(0)}{\partial p_{i}} & \mathbf{0}
\end{array}\right)\left(\begin{array}{l}
\left.\mathbf{A}_{1} \mathbf{V}_{1}^{H}\right) \\
\mathbf{U}_{2} \mathbf{A}_{2}
\end{array}\right) \mathbf{A}_{2} \\
& \quad=-\mathbf{V}_{1}^{H}\left(\frac{\partial \mathbf{M}(0)}{\partial p_{i}} \mathbf{U}_{2} \mathbf{A}_{2}^{2}+\frac{\partial \mathbf{C}(0)}{\partial p_{i}} \mathbf{U}_{2} \mathbf{A}_{2}+\frac{\partial \mathbf{K}(0)}{\partial p_{i}} \mathbf{U}_{2}\right)
\end{aligned}
$$

we have:

$\frac{\partial \mathbf{V}_{1}^{H}(0)}{\partial p_{i}}=\Theta_{2}^{-1}\left[-\mathbf{V}_{1}^{H}\left(\frac{\partial \mathbf{M}(0)}{\partial p_{i}} \mathbf{U}_{2} \mathbf{A}_{2}^{2}+\frac{\partial \mathbf{C}(0)}{\partial p_{i}} \mathbf{U}_{2} \mathbf{A}_{2}+\frac{\partial \mathbf{K}(0)}{\partial p_{i}} \mathbf{U}_{2}\right)\right] \mathbf{V}_{2}^{H}-\mathbf{V}_{1}^{H} \frac{\partial \mathbf{M}(0)}{\partial p_{i}} \mathbf{M}^{-1}(0)$

So the proof of the theorem is completed.

\section{An example}

Let $p=\left(p_{1}, p_{2}\right)$, consider the matrix, consider the matrix:

$\mathbf{A}(p)=\left(\begin{array}{ccc}2 p_{1}+p_{2}+1 & p_{1}+p_{2}+1 & p_{1} \\ 2 p_{2} & p_{1}+1 & p_{2} \\ p_{1} & p_{2} & 0\end{array}\right), \quad \mathbf{B}(p)=\left(\begin{array}{ccc}1 & p_{1} & 0 \\ 0 & 1 & 0 \\ 0 & 0 & 1\end{array}\right)$

Then:

$\mathbf{A}(0)=\left(\begin{array}{lll}1 & 1 & 0 \\ 0 & 1 & 0 \\ 0 & 0 & 0\end{array}\right), \quad \mathbf{B}(p)=\left(\begin{array}{lll}1 & 0 & 0 \\ 0 & 1 & 0 \\ 0 & 0 & 1\end{array}\right)$

The eigenvalue of $\{\mathbf{A}(0), \mathbf{B}(0)\}$ is $\lambda=1$ with multiplicity $r=2$. The derivatives of the matrices are:

$\frac{\partial \mathbf{A}(0)}{\partial p_{1}}=\left(\begin{array}{lll}2 & 1 & 1 \\ 0 & 1 & 0 \\ 1 & 0 & 0\end{array}\right), \quad \frac{\partial \mathbf{B}(0)}{\partial p_{1}}=\left(\begin{array}{lll}0 & 1 & 0 \\ 0 & 0 & 0 \\ 0 & 0 & 0\end{array}\right)$
$\frac{\partial \mathbf{A}(0)}{\partial p_{2}}=\left(\begin{array}{lll}1 & 1 & 0 \\ 2 & 0 & 1 \\ 0 & 1 & 0\end{array}\right)$,

Let $\mathbf{X}=\mathbf{I}_{3}$ and $\mathbf{Y}=\mathbf{I}_{3}$, then: 
$\mathbf{Y}^{H} \mathbf{A}(0) \mathbf{X}=\left(\begin{array}{lll}1 & 1 & 0 \\ 0 & 1 & 0 \\ 0 & 0 & 1\end{array}\right), \quad \mathbf{Y}^{H} \mathbf{B}(0) \mathbf{X}=\left(\begin{array}{lll}1 & 0 & 0 \\ 0 & 1 & 0 \\ 0 & 0 & 1\end{array}\right)$

From Theorem 1 we have:

$\frac{\partial \lambda_{\text {aver }}(0)}{\partial p_{1}}=\frac{1}{2} \operatorname{tr}\left[\left(\begin{array}{ll}2 & 1 \\ 0 & 1\end{array}\right)-\left(\begin{array}{ll}0 & 1 \\ 0 & 0\end{array}\right)\left(\begin{array}{ll}1 & 1 \\ 0 & 1\end{array}\right)\right]=\frac{1}{2} \operatorname{tr}\left(\begin{array}{ll}2 & 0 \\ 0 & 1\end{array}\right)=\frac{3}{2}$,
$\frac{\partial \lambda_{\text {aver }}(0)}{\partial p_{2}}=\frac{1}{2} \operatorname{tr}\left[\left(\begin{array}{ll}1 & 1 \\ 2 & 0\end{array}\right)-\left(\begin{array}{ll}0 & 0 \\ 0 & 0\end{array}\right)\left(\begin{array}{ll}1 & 1 \\ 0 & 1\end{array}\right)\right]=\frac{1}{2} \operatorname{tr}\left(\begin{array}{ll}1 & 1 \\ 2 & 0\end{array}\right)=\frac{1}{2}$,
$\frac{\partial \mathbf{X}_{1}(0)}{\partial p_{1}}=\left(\begin{array}{l}0 \\ 0 \\ 1\end{array}\right) \Theta_{1}^{-1}\left(\begin{array}{ll}1 & 0\end{array}\right)=\left(\begin{array}{l}0 \\ 0 \\ 1\end{array}\right)\left(\begin{array}{cc}1 & -1\end{array}\right)=\left(\begin{array}{cc}0 & 0 \\ 0 & 0 \\ 1 & -1\end{array}\right)$,
$\frac{\partial \mathbf{X}_{1}(0)}{\partial p_{2}}=\left(\begin{array}{l}0 \\ 0 \\ 1\end{array}\right) \Theta_{1}^{-1}\left(\begin{array}{ll}0 & 1\end{array}\right)=\left(\begin{array}{l}0 \\ 0 \\ 1\end{array}\right)\left(\begin{array}{ll}0 & 1\end{array}\right)=\left(\begin{array}{ll}0 & 0 \\ 0 & 0 \\ 0 & 1\end{array}\right)$,
$\frac{\partial \mathbf{X}_{2}(0)}{\partial p_{1}}=\Theta_{2}^{-1}\left(\begin{array}{l}0 \\ 1\end{array}\right)\left(\begin{array}{lll}0 & 0 & 1\end{array}\right)-\left(\begin{array}{lll}0 & 1 & 0 \\ 0 & 0 & 0\end{array}\right)=\left(\begin{array}{lll}0 & -1 & 1 \\ 0 & 0 & 0\end{array}\right)$,
$\frac{\partial \mathbf{X}_{2}(0)}{\partial p_{2}}=\Theta_{2}^{-1}\left(\begin{array}{l}0 \\ 1\end{array}\right)\left(\begin{array}{lll}0 & 0 & 1\end{array}\right)=\left(\begin{array}{lll}0 & 0 & -1 \\ 0 & 0 & 1\end{array}\right)$.

\section{Conclusion}

The derivatives of eigenvalues and eigenvectors, which characterize the tendency of variation for frequencies and mode shapes with respect to design parameters, are widely used in many applications. For example, one of the most commonly used methods in damage detection of bridge is vibration test analysis method, whose idea is to discrete bridge by finite element method or finite difference method and convert the dynamic model of the bridge structure to computing the eigensensitivity of a damped system. This paper proposes the theoretical analysis on sensitivity of defective multiple eigenvalues. For generalized eigenvalue problem, the derivatives of the average eigenvalues and the corresponding eigenvector matrices (Theorem 1) are both obtained by the equivalence of generalized eigenvalue problem and standard eigenvalue problem when mass matrix is invertible. For quadratic eigenvalue problem, we can translate quadratic eigenvalue problem into generalized eigenvalue problem, then by the result of generalized eigenvalue problem, the average of eigenvalues is proved to be analytic, the derivatives of the average eigenvalues and the corresponding eigenvector matrices (Theorem 3 ) are obtained when the mass matrix is invertible.

\section{Acknowledgements}

This work was supported by National Natural Science Foundation of China (Nos. 61503160 and 61572242), and Natural Science Foundation of the Jiangsu Higher Education Institutions of China (No. 15KJB110004). The authors are grateful to the referees for their valuable comments and suggestions which helped to improve the presentation of this paper.

\section{References}

[1] Haug E. J., Choi K. K., Komkov V. Design Sensitivity Analysis of Structural Systems. Academic Press, New York 1986.

[2] Haftka R. T., Adelman H. M. Recent developments in structural sensitivity analysis. Structural Optimization, Vol. 1, Issue 3, 1989, p. 137-151.

[3] Mottershead J. E., Friswell M. I. Model updating in structural dynamics: a survey. Journal of Sound and Vibration, Vol. 167, 1993, p. 347-375. 
[4] Friswell M. I., Mottershead J. E. Finite Element Model Updating in Structural Dynamics. Kluwer Academic, Norwell, MA, 1995.

[5] Messina A., Williams E. J., Contursi T. Structural damage detection by a sensitivity and statistical-based method. Journal of Sound and Vibration, Vol. 216, 1998, p. 791-808.

[6] Lancaster P. On eigenvalues of matrices dependent on a parameter. Numerische Mathematik, Vol. 6, 1964, p. 377-387.

[7] Kato T. Perturbation Theory for Linear Operators. Spring-Verlag, New York, 1966.

[8] Rellich F. Perturbation Theory of Eigenvalue Problems. Gordan and Breach, New York, 1969.

[9] Sun J. G. Eigenvalues and eigenvectors of a matrix dependent on several parameters. Journal of Computational Mathematics, Vol. 3, Issue 4, 1985, p. 351-364.

[10] Meyer C. D., Stewart G. W. Derivatives and perturbations of eigenvectors. SIAM Journal on Numerical Analysis, Vol. 25, 1988, p. 679-691.

[11] Lancaster P., Markus A. S., Zhou F. Perturbation theory for analytic matrix functions: the semisimple case. SIAM Journal on Matrix Analysis and Applications, Vol. 25, 2003, p. 606-626.

[12] Sun J. G. A note on local behavior of multiple eigenvalues. SIAM Journal on Matrix Analysis and Applications, Vol. 10, 1989, p. 533-541.

[13] Sun J. G. Multiple eigenvalue sensitivity analysis. Linear Algebra and its Applications, Vols. 137-138, 1990, p. 183-211.

[14] Chu K.-W.E. On multiple eigenvalues of matrices depending on several parameters. SIAM Journal on Numerical Analysis, Vol. 27, 1990, p. 1368-1385.

[15] Xie H. Q., Dai H. On the sensitivity of multiple eigenvalues of nonsymmetric matrix pencils. Linear Algebra and its Applications, Vol. 374, 2003, p. 143-158.

[16] Andrew A. L., Chu K.-W. E., Lancaster P. Derivatives of eigenvalues and eigenvectors of matrix functions. SIAM Journal on Matrix Analysis and Applications, Vol. 14, 1993, p. 903-926.

[17] Fox R. L., Kapoor M. P. Rates of changes of eigenvalues and eigenvectors. AIAA Journal, Vol. 6, 1968, p. 2426-2429.

[18] Adhikari S. Derivative of eigensolutions of nonviscously damped linear systems. AIAA Journal, Vol. 40, 2002, p. 2061-2069.

[19] Adhikari S. Calculation of derivative of complex modes using classical normal modes. Computers and Structures, Vol. 77, 2000, p. 625-633.

[20] Nelson R. B. Simplified calculation of eigenvector derivatives. AIAA Journal, Vol. 14, 1976, p. 1201-1205.

[21] Friswell M. I., Adhikari S. Derivatives of complex eigenvectors using Nelsons method. AIAA Journal, Vol. 38, 2000, p. 2355-2357.

[22] Guedria N., Chouchane M., Smaoui H. Second-order eigensensitivity analysis of asymmetric damped systems using Nelson's method. Journal of Sound and Vibration, Vol. 300, 2007, p. 974-992.

[23] Adhikari Friswell S. M. I. Calculation of eigen solution derivatives for nonviscously damped systems using Nelsons method. AIAA Journal, Vol. 44, 2006, p. 1799-1806.

[24] Wang P. X., Dai H. Calculation of eigenpair derivatives for asymmetric damped systems with distinct and repeated eigenvalues. International Journal for Numerical Methods in Engineering, Vol. 103, 2015, p. 501-515.

[25] Omenzetter P. Sensitivity analysis of the eigenvalue problem for general dynamic systems with application to bridge deckflutter. Journal of Engineering Mechanics, Vol. 138, 2012, p. 675-682.

[26] Rogers L. C. Derivatives of eigenvalues and eigenvectors. AIAA Journal, Vol. 8, 1970, p. 943-944.

[27] Plaut R. H., Huseyin K. Derivatives of eigenvalues and eigenvectors in non-self-adjoint systems. AIAA Journal, Vol. 11, 1973, p. 250-251.

[28] Garg S. Derivatives of eigensolutions for a general matrix. AIAA Journal, Vol. 11, 1973, p. 1191-1194.

[29] Rudisill C. S. Derivatives of eigenvalues and eigenvectors for a general matrix. AIAA Journal, Vol. 12, 1974, p. 721-722.

[30] Rudisill C. S., Chu Y. Numerical methods for evaluating the derivatives of eigenvalues and eigenvectors. AIAA Journal, Vol. 13, 1975, p. 834-837.

[31] Lee I. W., Jung J. H. An efficient algebraic method for the computation of natural frequency and mode shape sensitivities: Part 1, distinct natural frequencies. Computers and Structures Vol. 62, 1997, p. $429-435$.

[32] Lee I. W., Kim D. O., Jung J. H. Natural frequency and mode shape sensitivities of damped systems: Part 1, distinct natural frequencies. Journal of Sound and Vibration, Vol. 223, 1999, p. 399-412. 
[33] Ojalvo I. U. Efficient computation of modal sensitivities for systems with repeated frequencies. AIAA Journal, Vol. 26, 1988, p. 361-366.

[34] Dailly R. L. Eigenvectors derivatives with repeated eigenvalues. AIAA Journal, Vol. 27, 1989, p. 486-491.

[35] Lee I. W., Jung J. H. An efficient algebraic method for the computation of natural frequency and mode shape sensitivities: Part 2, multiple natural frequencies. Computers and Structures, Vol. 62, 1997, p. 437-443.

[36] Andrew A. L., Tan R. C. E. Computation of derivatives of repeated eigenvalues and the corresponding eigenvectors of symmetric matrix pencils. SIAM Journal on Matrix Analysis and Applications, Vol. 20, 1989, p. 78-100.

[37] Lee I. W., Kim D. O., Jung J. H. Natural frequency and mode shape sensitivities of damped systems: Part 2, Multiple natural frequencies. Journal of Sound and Vibration, Vol. 223, 1999, p. 413-424.

[38] Tang J., Ni W. M., Wang W. L. Eigensolutions sensitivity for quadratic eigenproblems. Journal of Sound and Vibration, Vol. 196, 1996, p. 179-188.

[39] Xie H. Q., Dai H. Derivatives of repeated eigenvalues and corresponding eigenvectors of damped systems. Applied Mathematics and Mechanics (English Edition), Vol. 28, 2007, p. 837-845.

[40] Xie H. Q., Dai H. Calculation of derivatives of multiple eigenpairs of unsymmetrical quadratic eigenvalue problems. International Journal of Computer Mathematics, Vol. 85, 2008, p. 1815-1831.

[41] Wang P. X., Dai H. Eigensensitivity analysis for symmetric nonviscously damped systems with repeated eigenvalues. Journal of Vibroengineering, Vol. 16, 2014, p. 4065-4076.

[42] Li L., Hu Y. J., Wang X. L. A study on design sensitivity analysis for general nonlinear eigenproblems. Mechanical Systems and Signal Processing, Vol. 34, 2013, p. 88-105.

[43] Qian J., Andrew A. L., Chu D., Tan R. C. E. Computing derivatives of repeated eigenvalues and corresponding eigenvectors of quadratic eigenvalue problems. SIAM Journal on Matrix Analysis and Applications, Vol. 34, 2013, p. 1089-1111.

[44] Adhikari S. Structural Dynamics with Generalized Damping Models: Analysis. Wiley-ISTE, 2013.

[45] Adhikari S. Structural Dynamics with Generalized Damping Models: Identification. Wiley-ISTE, 2013.

[46] Bochner S., Martin W. T. Several Complex Variables. Princeton University Press, Princeton, 1948.

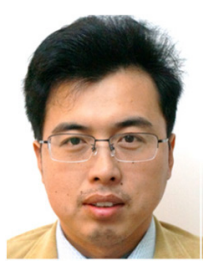

Pingxin Wang received the Master's degree in Nanjing University of Science and Technology, Nanjing, Chin, and his Ph.D. degree in Computational Mathematics from Nanjing University of Aeronautics and Astronautics (NUAA), Nangjing, China. His current research interests include matrix analysis and sensitivity analysis.

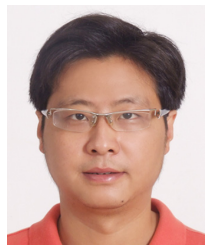

Xibei Yang received the Master's degree in Jiangsu University of Science and Technology, Zhenjiang, China, and his Ph.D. degree in Computer Science and Engineering from Nanjing University of Science and Technology Nangjing, China. His current research interests include granular computing and rough set. 